\title{
LEUKOCYTOCLASTIC VASCULITIS AND THROMBOSIS IN PRIMARY SJÖGREN'S SYNDROME AFTER COVID-19: A CASE REPORT
}

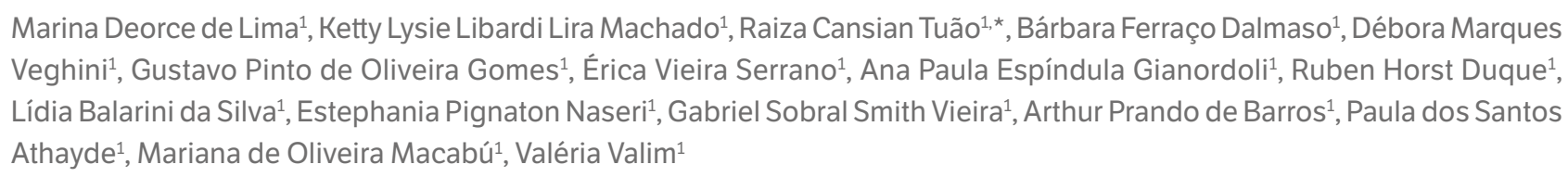

1.Universidade Federal do Espírito Santo, Vitória (ES), Brazil.

*Corresponding author: raizatuao@hotmail.com

\section{BACKGROUND}

Primary Sjögren's syndrome (pSS) is a systemic autoimmune disease characterized by inflammation of the lacrimal and salivary glands, in addition to extraglandular manifestations, including constitutional symptoms and specific organ involvement. In parallel, COVID-19 presents itself as an infectious disease that can trigger an abnormal immune response. Leukocytoclastic vasculitis (LCV), caused by the deposition of immune complexes on the vessel walls and activation of the complement system, may be a reaction both to COVID-19 infection and secondary to an existing systemic autoimmune disease. This study aims to report a case of pSS with LCV and small and medium vessel thrombosis that occurred after a COVID-19 event.

\section{CASE REPORT}

Female patient, white, 62 years old, diagnosed with pSS since 2017, with xerostomia and xerophthalmia, altered Schirmer test and salivary flow rate, salivary gland biopsy with eight inflammatory foci in $12 \mathrm{~mm} 2$ (focus-score: 2.6) anti-SSA > 240, and positive anticardiolipin IgG. She had been using azathioprine since December 2020, maintaining remission, when she presented COVID-19 infection in March 2021 (confirmed by RT-PCR test). After 15 days, she started polyarthralgia, worse in the ankle and left hand, and increased C-reactive protein (CRP $367 \mathrm{mg} / \mathrm{L}$ ). In April 2021, she developed hemicranial headache, photophobia, conjunctival hyperemia and bilateral reduction in visual acuity. Fundoscopy revealed glaucoma secondary to bilateral anterior uveitis, anterior scleritis in the left eye and posterior scleritis in both eyes, secondary to pSS activity. Pulse therapy with methylprednisolone and cyclophosphamide was instituted, with partial improvement in visual acuity. After 10 days, the patient started cyanosis and edema of the lower limbs, evolving with dry gangrene in the 1st to 5th left toes and 2nd and 3rd right toes, requiring amputation of the left forefoot and 2nd right toe. The pathological examination showed neutrophilic vasculitis with leukocytoclasia and thrombosis of small and medium arteries. The patient was discharged from the hospital for outpatient follow-up, maintaining pulse therapy with cyclophosphamide.

\section{CONCLUSION}

Although the correlation between COVID-19 and autoimmune diseases is not completely clear, it is known that viral infections can disrupt immune tolerance and trigger autoimmune responses. This association was essential for the development of complications in the patient. Faced with COVID-19 infection in patients with autoimmune diseases, one should pay attention to complications such as vasculitis and thrombosis, in addition to thromboembolic events.

\section{KEYWORDS}

Autoimmune diseases, Sjögren's syndrome, Scleritis, Thrombosis. 